\title{
A Low Complexity Iterative Receiver based on Successive Cancellation for MIMO
}

\author{
Holger Claussen ', Hamid Reza Karimi ${ }^{2}$, Bernard Mulgrew 1 \\ ' Signals \& Systems Group, University of Edinburgh, UK \\ ${ }^{2}$ Bell Labs Research, Lucent Technologies, Swindon, UK
}

\begin{abstract}
Turbo-encoded multiple-input multiple-output (MIMO) systems have recently been proposed for the support of high-speed downlink packet access (HSDPA) in UMTS, where the re-use of spreading codes across the transmitter antennas results in high levels of interference. The state of the art receiver for this system incorporates a channel equalizer, followed by an a posteriori probability (APP) detector and a turbo decoder. However, the complexity of APP detection can become prohibitive since it grows exponentially with the number of transmitter antennas and the modulation order. In this paper, a MIMO receiver is proposed which replaces the optimum but complex APP detector by successive interference cancellation (SIC) incorporating sub-optimal matched filter detection. Using convolutional encoding at the transmitter, the receiver performance is sustained via iterations between the simplified detector and the convolutional decoder. In combination with a proposed novel soft-output combining scheme, it is shown that the new receiver can outperform the APP-based receiver at a much lower complexity and with no need for channel equalization.
\end{abstract}

Key words: Iterative detection, MIMO, successive cancellation, serial interference cancellation, SIC, soft output combining, order metric, ordering

\section{INTRODUCTION}

Turbo-encoded multiple-input multiple-output (MIMO) systems have recently been proposed for the support of high-speed downlink packet access (HSDPA) in UMTS [1]. The concept here is to increase the achievable data rates for a particular user through a combination of code re-use across transmit antennas and higher-order modulation schemes. The code re-use inevitably results in high levels of interference at the mobile receiver, even under non-dispersive channel conditions. In order to tackle such high interference levels, receivers based on the optimal a posteriori probability (APP) detector [2] followed by turbo decoding have been proposed [3][4]. To cope with dispersive channels and in order to avoid sequence estimation, it is necessary to use an APP detector preceded by a matrix channel equaliser.

Essentially, the APP detector operates by computing soft-outputs for the transmitted bits which most closely match the received signal in an Euclidian sense. The

The original version of this chapter was revised: The copyright line was incorrect. This has been corrected. The Erratum to this chapter is available at DOI: 10.1007/978-0-387-35618-1_37 


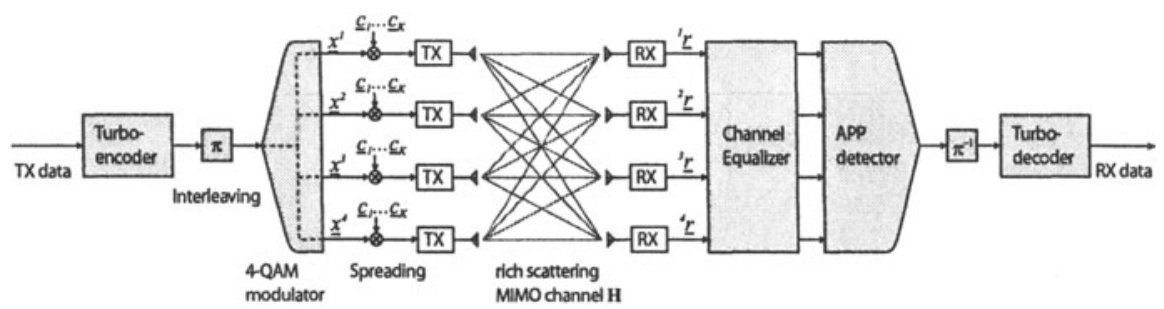

Figure 1. Equalized APP based receiver for a $4 \times 4$ MIMO system (Reference)

computational complexity of the APP detector is an exponential function of the total number of bits transmitted during a symbol epoch, which is equal to the product of the number of transmit antennas and the number of bits per symbol. Consequently, the complexity of the APP detector can become prohibitive for increasing numbers of transmit antennas and (perhaps more importantly) modulation orders. This inflexibility of the optimal APP detector has resulted in renewed interest in the use of suboptimal but less complex MIMO detectors.

Successive interference cancellation (SIC) schemes have been considered for many years in the context of multi-user detection for the CDMA uplink [5][6][7]. These schemes combat interference by successively detecting and cancelling the influence of data streams from the received signal. The more reliable data streams are detected and cancelled first. In the context of MIMO receivers, the original BLAST detector [8] is essentially a SIC architecture incorporating ordering and detection based on the minimum mean-squared error (MMSE) criterion. Furthermore, significant performance improvements have been demonstrated through iterations between the BLAST detector and a convolutional decoder [9].

In this paper, a bit-based SIC scheme incorporating simple matched filters (MF) as the basic detection unit is considered as a receiver for a convolutionally-encoded MIMO link. The MF-SIC detector performs iterations with a convolutional decoder in conjunction with a novel soft-output combining technique. Convolutional coding is used, since it provides better convergence than turbo coding in iterative schemes. The combining acts to suppress instabilities caused by erroneously detected and cancelled bits. The resulting receiver architecture is highly scalable in terms of dealing with growing numbers of transmit antennas and high-order modulation schemes.

The proposed MF-SIC receiver is compared with the APP-based receiver considered for an equivalent turbo-encoded MIMO link [4] and is shown to achieve superior performance at a much lower complexity. The performance loss due to the use of a sub-optimal detector is regained via iterations with the decoder, enabled by the novel soft-output combining technique.

\section{SIGNAL MODEL}

Figures (1) and (2) illustrate the transmission scheme for the MIMO system under investigation. At the transmitter, user data is convolutional or turbo encoded and interleaved. The coded data stream is de-multiplexed into $N_{T}$ sub-streams, corresponding to the $N_{T}$ transmit antennas. Each sub-stream is then modulated on to $N K$ 4-QAM symbols and subsequently spread by a factor $Q$ via a set of $K$ orthogonal spreading codes prior to transmission. Each transmitted spread stream then occupies $N$ symbol 


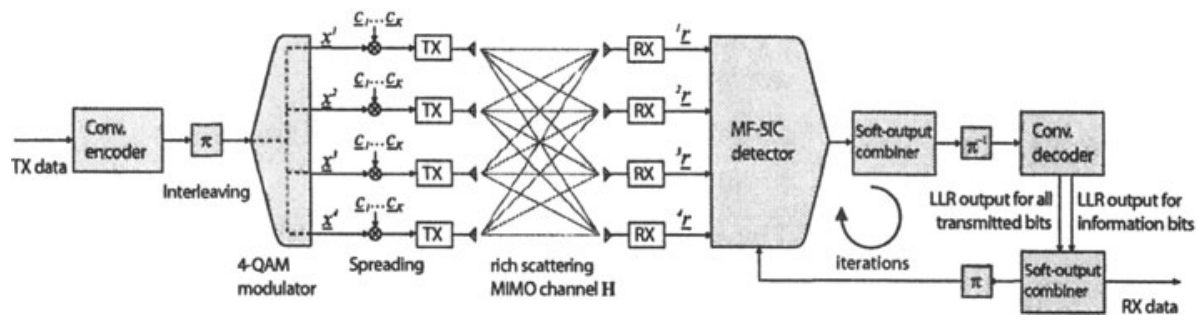

Figure 2. Iterative MF-SIC based receiver for a $4 \times 4$ MIMO system

intervals. Also note that the same set of $K$ codes are re-used across all transmit antennas. Therefore, the MIMO propagation environment, which is assumed to exhibit significant multipath, plays a major role in achieving signal separation at the receiver. The transmitted signals are received by $N_{R}$ receive antennas after propagation through dispersive radio channels with impulse response lengths of $W$ chips. The received signal vector can then be modelled as follows:

$$
\begin{aligned}
& {\left[\begin{array}{c}
{ }^{1} \underline{\underline{r}} \\
\vdots \\
{ }^{N_{R}} \underline{\underline{r}}
\end{array}\right]=\left[\begin{array}{ccc}
{ }^{1} \boldsymbol{H}^{1} & \ldots & { }^{1} \boldsymbol{H}^{N_{T}} \\
\vdots & \ddots & \vdots \\
{ }^{N_{R}} \boldsymbol{H}^{1} & \ldots & { }^{N_{R}} \boldsymbol{H}^{N_{T}}
\end{array}\right] \sum_{k=1}^{K}\left[\begin{array}{ccc}
C_{k}^{\prime} & \cdots & 0 \\
\vdots & \ddots & \vdots \\
0 & \cdots & C_{k}^{\prime}
\end{array}\right]\left[\begin{array}{c}
\underline{x}_{k}^{1} \\
\vdots \\
\underline{x}_{k}^{N_{T}}
\end{array}\right]+\left[\begin{array}{c}
{ }^{1} \underline{v} \\
\vdots \\
{ }^{N_{R}} \underline{v}
\end{array}\right]} \\
& \underline{\boldsymbol{H}} \sum_{k=1}^{K} \boldsymbol{C}_{k} \underline{x}_{k}+\underline{v}
\end{aligned}
$$

where: ${ }^{m} \underline{r} \in \mathrm{C}^{(Q N+W-1) \times 1} \quad$ Signal at Rx antenna $m$.

$m_{\underline{v}} \in C^{\left(Q^{N+W-1) \times 1}\right.} \quad$ Noise + inter-cell interference at $R x$ antenna $m$.

$\underline{x}_{k}^{n} \in \mathrm{C}^{N \times 1} \quad$ Symbol sequence $\left[x_{k}^{n}(1) \ldots x_{k}^{n}(N)\right]^{\mathrm{T}}$ at Tx antenna $n$ spread via $k^{\text {th }}$ spreading code.

$C_{k}^{\prime} \in \mathrm{C}^{Q N \times N} \quad$ Spreading matrix for $k^{\text {th }}$ spreading code $\underline{c}_{k} \in \mathrm{C}^{Q \times 1}$.

$$
C_{k}^{\prime}=\underbrace{\left[\begin{array}{ccc}
\underline{c}_{k} & \cdots & \underline{0} \\
\vdots & \ddots & \vdots \\
\underline{0} & \cdots & \underline{c}_{k}
\end{array}\right]}_{N \text { Times }}
$$

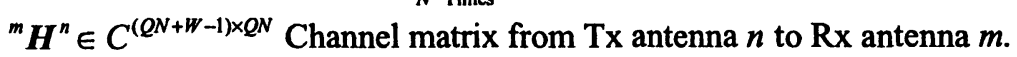

and $\underline{v}$ is a vector of iid complex Gaussian variables, $R_{v}=\mathrm{E}\left\{\underline{v v^{\mathrm{H}}}\right\}=N_{0} I$. The 4-QAM modulation mapping is such that $x_{k}^{n}(t)=b_{k, 0}^{n}(t)+j b_{k, 1}^{n}(t)$ with $b_{k, i}^{n}(t) \in\{+1,-1\}$.

\section{APP RECEIVER}

As indicated in Figure (1), in this receiver the signal vector $\underline{r}$ is applied to an $a$ posteriori probability (APP) detector following a process of channel equalization. The soft outputs from the APP detector are then applied to a turbo decoder which generates reliable estimates of the transmitted bits.

A full space-time APP detector implies joint detection of $K N_{T}$ transmitted symbols per symbol epoch. For 4-QAM modulation, and for dispersive channels with ISI extending over $L$ symbols, this requires a search over a trellis containing $2^{2(L+1) K N_{r}}$ states. 
The computational complexity is clearly inhibitive for typical parameter values. Note that, in flat fading conditions $(L=0)$ and for $K$ orthogonal codes re-used over the transmit antennas, the number of trellis states reduces to a more realistic value of $2^{2 N_{r}}$. As a result, a strategy for dealing with dispersive channels is to apply APP detection after a process of space-time equalization which effectively eliminates dispersion. The equalization process inevitably causes noise colouring, which needs to be accounted for in APP detection.

\section{Space-Time Equalization}

The received signal over $N$ symbol epochs may be written as

$$
\underline{r}=\boldsymbol{H} \sum_{k=1}^{K} \boldsymbol{C}_{k} \underline{x}_{k}+\underline{v}=\boldsymbol{H} \boldsymbol{C} \underline{x}+\underline{v}
$$

where $\underline{s}=C \underline{x}$ is the vector of spread symbols. A minimum mean-square error (MMSE) equalizer represents a space-time matrix $V$ which minimizes the term $\mathrm{E}\left\{\|\underline{\underline{S}}-\boldsymbol{V} \boldsymbol{r}\|^{2}\right\}$. It is easy to show [10], that the solution to this problem is given by

$$
\boldsymbol{V}=\boldsymbol{R}_{s} \boldsymbol{H}^{\mathrm{H}}\left(\boldsymbol{H} \boldsymbol{R}_{s} \boldsymbol{H}^{\mathrm{H}}+\boldsymbol{R}_{v}\right)^{-1}
$$

where $R_{s}=\mathrm{E}\left\{\underline{s s}^{\mathrm{H}}\right\}=2 C C^{\mathrm{H}}$ since $\mathrm{E}\left\{\underline{x x^{\mathrm{H}}}\right\}=2 I$. The equalization process $V \underline{r}$ essentially eliminates the effects of the channel matrix $H$. As a result, assuming orthogonal spreading codes, the contribution of symbols transmitted using the $k^{\text {th }}$ spreading code can be retrieved at the output of the equalizer via the de-spreading operation

$$
\underline{z}_{k}=C_{k}^{\mu} V \underline{r}=T_{k} \underline{r} \approx\left\|\underline{c}_{k}\right\|^{2} \underline{x}_{k}+T_{k} \underline{v} \quad \in \quad C^{N_{T} N \times 1} \quad \text { and } \quad k=1 \ldots K
$$

where $\underline{v}_{k}=\boldsymbol{T}_{k} \underline{\underline{v}}$ is coloured noise. Due to excessive computational complexity, space-time equalization is usually performed over a block of $N_{E}<N$ symbols and repeated $N / N_{E}$ times to cover the overall transmission period.

\section{APP Detection}

Vector $\underline{z}_{k}$ consists of the equalized and de-spread contributions of $N_{T} N$ symbols transmitted via the $k^{\text {th }}$ spreading code over a total of $N$ symbol epochs. Considering only the $N_{T}$ rows of Eq. (5) corresponding to the $t^{\text {th }}$ symbol epoch, we have

$$
\underline{z}_{k}(t)=\left\|\underline{c}_{k}\right\|^{2} \underline{x}_{k}(t)+T_{k}(t) \underline{v} \in \mathrm{C}^{N_{T} \times 1} \quad \text { and } \quad k=1 \ldots K
$$

The APP algorithm can then be applied to derive log-likelihood ratios for each (equally probable) transmitted bit, $b_{k, i}{ }^{n}(t) n=1 \ldots N_{T} i \in\{0,1\}$, in the form of soft outputs

$$
\begin{aligned}
& y\left(b_{k, i}^{n}(t)\right)=\min _{\underline{x}_{k}^{n}(t) b b_{k}^{n},(t)=-1}\left\{\left\|R_{u_{k}(t)}^{\frac{-1}{2}}\left(\underline{z}_{k}(t)-\left\|\underline{c}_{k}\right\|^{2} \underline{x}_{k}^{n}(t)\right)\right\|^{2}\right\} \\
& -\min _{\underline{x}_{k}^{n}(t) \mid b_{k}^{n},(t)=+1}\left\{\left\|R_{u_{k}(t)}^{\frac{-1}{2}}\left(\underline{z}_{k}(t)-\left\|\underline{c}_{k}\right\|^{2} \underline{x}_{k}^{n}(t)\right)\right\|^{2}\right\}
\end{aligned}
$$

where $\underline{u}_{k}(t)=\boldsymbol{T}_{k}(t) \underline{\underline{\nu}}$ is coloured noise and $\boldsymbol{R}_{u_{k}(t)}=\mathrm{E}\left\{\underline{u}_{k}(t) \underline{u}_{k}(t)^{\mathrm{H}}\right\}=N_{o} \boldsymbol{T}_{k}(t) \boldsymbol{T}_{k}(t)^{\mathrm{H}}$. The soft outputs are then applied to a Turbo decoder whose constituent decoders operate based on the max-log MAP algorithm. 


\section{ITERATIVE RECEIVER}

At the receiver of Figure (2), the signal vector $\underline{r}$ is fed into a successive interference canceller incorporating matched filter detection (MF-SIC). The received signal of Eq. (1) observed over the $t^{\text {th }}$ symbol epoch may be written as

$$
\underline{r}(t)=\sum_{n=1}^{N_{T}} \sum_{k=1}^{K} \underline{a}_{k}^{n}(t) x_{k}^{n}(t)+\mathrm{ISI}+\underline{v}(t) \in \mathrm{C}^{N_{k}(Q+W-1) \times 1}
$$

where $x_{k}^{n}(t)$ is a transmitted symbol at the $t^{\text {th }}$ symbol epoch and $\underline{a}_{k}^{n}(t)$ is its codechannel signature at the receiver. The output of the MF-SIC is then de-interleaved and applied to a convolutional decoder. This represents the first iteration of the receiver. Soft outputs from the decoder are then re-interleaved and applied to the MF-SIC for further iterations.

\section{Iteration 1:}

Here, the MF-SIC operates at a symbol level. The first step is to determine, at each symbol interval $t$, the most reliable symbol according to a reliability criterion. Ideally, the symbol with the lowest error probability is selected [6]. Lacking such information, the symbol $x_{k}^{n}(t) k=1 \ldots K n=1 \ldots N_{T}$ with the highest signature energy, $\left|\underline{a}_{k}^{n}(t)\right|^{2}$ (or least mean-square estimation error), is selected. The next step is to estimate the selected symbol (soft-output derived via matched filter detection), make a hard decision on the estimate, reconstruct and cancel its contribution from the received signal:

$$
\begin{aligned}
& y_{k}^{n}(t)=\underline{a}_{k}^{n}(t)^{\mathrm{H}} \underline{r}(t) \\
& \underline{r}(t)=\underline{r}(t)-\underline{a}_{k}^{n}(t)\left\{\operatorname{sgn}\left\{\operatorname{Re}\left[y_{k}^{n}(t)\right]\right\}+j \operatorname{sgn}\left\{\operatorname{Im}\left[y_{k}^{n}(t)\right]\right\}\right\}
\end{aligned}
$$

The process is then repeated for the next most reliable symbol. If the decision on the selected symbol is correct, then its interference towards other symbols can be completely suppressed. However, a wrong decision doubles the level of interference caused by the erroneously detected symbol. Consequently, the reliability criterion used for the ordering of symbols is of critical importance in any form of successive cancellation.

After the MF-SIC detection of a complete code-block, the corresponding soft-outputs, $y_{k, 0}{ }^{n}(t)=\operatorname{Re}\left[y_{k}{ }^{n}(t)\right]$ and $y_{k, 1}{ }^{n}(t)=\operatorname{Im}\left[y_{k}{ }^{n}(t)\right]$, are multiplexed into a single stream for de-interleaving and convolutional decoding (max-log MAP algorithm). The decoder output is fed into the soft-output combiner and an interleaver prior to re-application to the MF-SIC for subsequent iterations.

\section{Iteration 2 and beyond:}

In the second iteration of the receiver, the MF-SIC has access to reliability information at a bit level, in the form of log-likelihood ratios, $\Lambda\left(b_{k, i}{ }^{n}(t)\right)$, generated by the soft-output decoder in the previous iteration. As a result, at each symbol interval $t$, ordering can be performed at a bit level (rather than symbol level) based on the loglikelihood ratios (LLRs). In other words, the bit $b_{k, i}{ }^{n}(t)$ with the largest LLR value $\left|\Lambda\left(b_{k, i}{ }^{n}(t)\right)\right|$ (or minimum estimation error probability), can be selected as most reliable. Since bit estimates corresponding to a particular symbol can have different reliabilities, the use of LLR values represents an optimum ordering policy. The cancellation 
process at the $t^{\text {th }}$ symbol interval is based on the more reliable hard bit estimates derived from the LLR values:

$$
\begin{aligned}
& y_{k, i}^{n}(t)=\frac{1}{2 j^{i}}\left\{\underline{a}_{k}^{n}(t)^{\mathrm{H}} \underline{r}(t)+(-1)^{i} \underline{r}(t)^{\mathrm{H}} \underline{a}_{k}^{n}(t)\right\} \\
& \underline{r}(t)=\underline{r}(t)-j^{i} \underline{a}_{k}^{n}(t) \operatorname{sgn}\left\{\Lambda\left(b_{k, i}^{n}(t)\right)\right\}
\end{aligned}
$$

where $i=0$ or 1 depending on whether the bit of interest forms the real or imaginary part of the 4-QAM symbol. The process is again repeated for the next most reliable bit. After the MF-SIC detection of a complete code-block, the soft-outputs $y$, are again multiplexed into a single stream for de-interleaving and decoding (max-log MAP algorithm). The performance of the MF-SIC (and hence the receiver) should improve at each iteration as the quality of the decoder output improves.

\section{Soft-Output Combining}

In the proposed iterative receiver, mutual information is exchanged between the MF-SIC detector and the convolutional decoder. Therefore, at each iteration, soft estimates (in the form of LLR values) at the output of the decoder are fed back to the detector for purposes of interference cancellation. Consequently, new and hopefully more reliable soft-output values are made available at the output of the decoder after each iteration. However, in some cases, the interference cancellation process can lead to poorer soft-outputs for certain bits. This can result in error propagation and therefore unstable bit-error rate performance in subsequent iterations.

Such instabilities can be avoided by combining the soft-output values computed in the current iteration with those computed in the previous iteration(s). The combining weight factors have a significant influence on the stability and the speed of convergence of the iterative receiver. Using this combining process, reliability information already gained for a certain transmitted bit is not lost in the next iteration.

While soft-output combining can be performed either at the output of the detector or that of the decoder, simulations indicate that a combination of both is most effective. If $q$ indicates the iteration index, then soft-output combining may be described as

$$
\begin{aligned}
& y_{k, i}^{n}(t)[q]=\alpha y_{k, i}^{n}(t)[q]+(1-\alpha) y_{k, i}^{n}(t)[q-1] \\
& \lambda_{k, i}^{n}(t)[q]=\beta \Lambda_{k, i}^{n}(t)[q]+(1-\beta) \Lambda_{k, i}^{n}(t)[q-1]
\end{aligned}
$$

Good performance results were found to be achieved via combining factors of $\alpha=0.9$ and $\beta=0.75$. Soft-output combining is an essential element of the proposed iterative receiver.

\section{PERFORMANCE AND COMPLEXITY COMPARISON}

The performance of the APP-based receiver for a Turbo-encoded MIMO link [4] is considered as reference for comparison with that of the proposed MF-SIC based receiver for an equivalent convolutionally-encoded MIMO link.

A system with $N_{T}=N_{R}=4, Q=16$ and $K=16$, similar to the HSDPA specifications is considered. In addition to a flat Rayleigh fading channel, a dispersive channel with 3 equal-power, chip-spaced taps is also considered. The assumed mobile speed is 3 


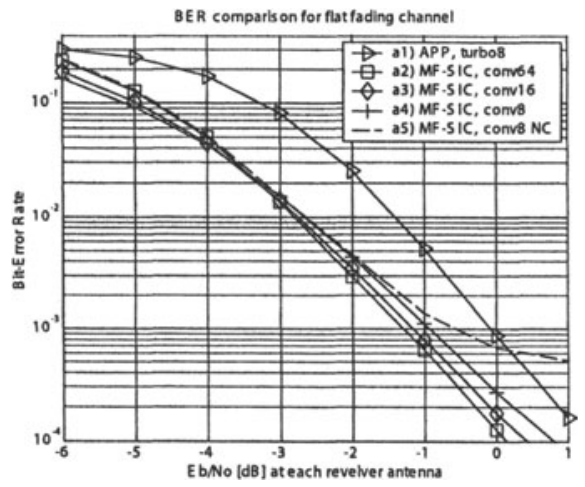

(a)

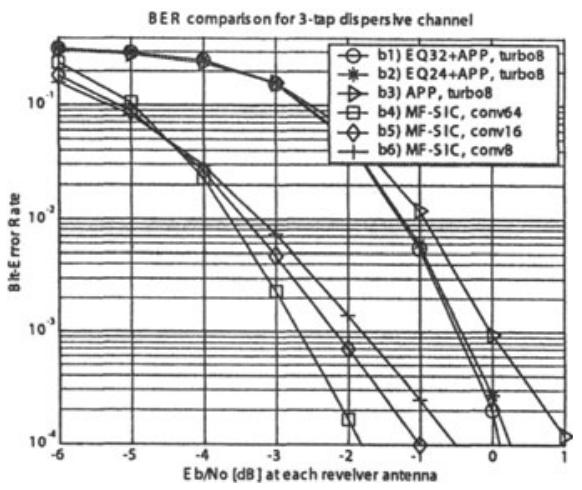

(b)

Figure 3. Performance comparison

$\mathrm{km} / \mathrm{h}$ and the receiver has perfect knowledge of the average channel conditions during each transmitted data block. For the turbo-encoded MIMO link, a 8-state rate 1/3 turbo encoder is used in accordance with the HSDPA specifications, resulting in a block size with up to 5114 information bits. A total of 6 iterations of the turbo decoder are performed in the receiver. For the convolutionally-encoded MIMO link, rate $1 / 3$, 8- 16- and 64-state convolutional encoders are considered to allow a comprehensive comparison in terms of performance and complexity. A total of 4 iterations between the MF-SIC detector and the convolutional decoder are performed. Soft-output combiners with the coefficients $\alpha=0.9$ after detection and $\beta=0.75$ after decoding were used.

The BER performance comparison is presented in Figure (3) for flat (a) and dispersive (b) channels, while Figure (4) illustrates the corresponding computational complexities in terms of the number of real multiplications.

For flat fading channels, the proposed iterative MF-SIC based receiver outperforms the APP-based receiver by approximately $1 \mathrm{~dB}$, dependent on the memory size of the convolutional code, consistently at a lower total computational complexity (a24). Simulation result (a5) clearly demonstrates the degradation in performance when soft-output combining is not used.

For the dispersive channel, the MF-SIC detectors offer again significant improvements in BER. In fact, the performance improvement over the equalized APP reference is even higher than for flat fading (result b4-6). Even with a simple 8-state convolutional decoder (b6), the proposed receiver offers improved BER results at approximately only $20 \%$ of the APP-based receiver complexity. The small performance differences for the equalized APP detector, between using equalizer block sizes of 32 or 24 chips (b2, b1), shows that the edge effects are negligible for this channel.
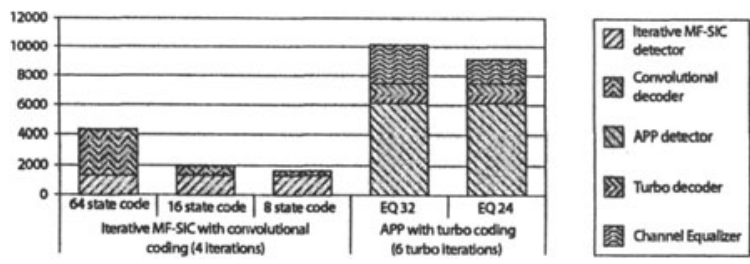

Figure 4. Complexity comparison: Multiplications per information bit 


\section{CONCLUSIONS}

In this paper, a low complexity iterative receiver for a convolutionally-encoded MIMO system is proposed and compared with an APP-based receiver for an equivalent turbo-encoded MIMO system. The proposed iterative receiver utilizes a successive interference cancellation architecture based on simple matched filters (MF-SIC). Despite the use of a simplified detection scheme, a high level of receiver performance is achieved via iterations with the decoder. In fact, it is shown that, via a combination of bit-based ordering/detection/cancellation and the use of a novel soft-output combining technique at the decoder output, the proposed low-complexity iterative receiver outperforms the APP-based receiver. Depending on the complexity of the convolutional decoder, the BER performance can be improved by up to $2 \mathrm{~dB}$, always at a significantly lower computational complexity. In contrast to the APP detector where the computational complexity grows exponentially with the number of antennas and the order of the modulation scheme, the complexity of the proposed iterative solution only grows linearly. This makes the proposed solution highly scalable and even more attractive for 16- and 64-QAM. Furthermore, unlike the APP-based receiver, the proposed solution does not require a matrix-channel equalizer to cope with dispersive propagation environments, making it attractive from an implementation standpoint. Finally, the iterative concept can be exploited to improve the channel estimation, which can further improve the performance in comparison to a non-iterative APP detector.

\section{REFERENCES}

[1] 3GPP TSG RAN WG1, "PARC with APP Decoding for HSDPA", TSG-R1(02)0549, April 2002, Paris, France.

[2] Benedetto, S.; Divsalar, D.; Montorsi, G.; Pollara, F., "A Soft Input Soft-Output APP Module for Iterative Decoding of Concntrated Codes", IEEE Communictions Letters, Volume 1, Issue 1, pp. 22 -24, January 1997.

[3] 3GPP TSG RAN WG1, "Further link level results for HSDPA using multiple antennas", TSG-RI\#17(00)1386, November 2000, Stockholm, Sweden.

[4] 3GPP TSG RAN WG1, "Link Level Results for HSDPA using Multiple Antennas in Correlated and Measured Channels", TSG-1\#19(01)0302. February 2001, Las Vegas, U.S.A.

[5] Verdu S., "Multiuser Detection", Cambridge University Press, 1998.

[6] Claussen, H.; Mulgrew, B; Karimi, H.R., "Performance Optimization of Successive Cancellation Detectors", World Wireless Congress, pp.797-802, May 2002, San Francicso, U.S.A.

[7] Guinand, P.S.; Kerr, R.W.; Moher, M., "Serial Interference Cancellation for Highly Correlated Users", IEEE Pacific Rim Conference on Communications, Computers and Signal Processing, pp. 133-136, August 1999, Victoria, Canada.

[8] Foschini G.J., "Layered Space-TimeArchitecture for Wireless Communication in a Fading Environment when using Multielement Antennas", Bell Labs Technical Journal, pp. 4159, Autumn 1996.

[9] Li, X.; Huang, H.; Foschini, G.J.; Valenzuela, R.A., "Effects of Iterative Detection and Decoding on the Performance of BLAST", GLOBECOM '00. Volume 2, pp. 1061-1066, November 2000, San Francisco, U.S.A.

[10] Klein, A.; Kaleh, G.K.; Baier, P.W., "Zero Forcing and Minimum Mean-Square-Error Equalization for Multiuser Detection in Code-Division Multiple-Access Channels", IEEE Transactions on Vehicular Technology, Volume 45, No. 2, May 1996. 